\title{
Sequential Release of Small Extracellular Vesicles from Bilayered Thiolated Alginate/Polyethylene Glycol Diacrylate Hydrogels for Scarless Wound Healing
}

Yifan Shen ${ }^{1 \#}$, Guanzhe Xu ${ }^{2,3 \#}$, Huanxuan Huang ${ }^{2 \#}$, Kaiyang Wang $^{1}$, Hui Wang ${ }^{4}$, Meidong Lang ${ }^{2 *}$,Hong $\mathrm{Gao}^{1^{*}}$, Shichang Zhao ${ }^{1^{*}}$

${ }^{1}$ Department of Orthopaedic Surgery, Shanghai Jiao Tong University Affiliated Sixth People’s Hospital, Shanghai 200233, China

${ }^{2}$ Shanghai Key Laboratory of Advanced Polymeric Materials, Key Laboratory for Ultrafine Materials of Ministry of Education, School of Materials Science and Engineering, East China University of Science and Technology, Shanghai 200237, China

${ }^{3}$ Internet of Things Research Center, Advanced Institute of Information Technology, Peking University, Hangzhou 311200, China

${ }^{4}$ Green Chemical Engineering Technology Research Center, Shanghai Advanced Research Institute, Chinese Academy of Sciences, Shanghai 201210, China. 


\section{Additional experimental methods}

Cells and cell culture: Human bone marrow-derived mesenchymal stem cells (hBMSCs; SCSP-405, Stem Cell Bank, Chinese Academy of Sciences) were cultured in minimum essential medium $\alpha$ ( $\alpha$-MEM; Gibco) supplemented with $10 \%$ fetal bovine serum (FBS; Gibco), penicillin $(100 \mathrm{U} / \mathrm{mL})$ and streptomycin $(100 \mu \mathrm{g} / \mathrm{mL})$ (Gibco) in an incubator with $5 \% \mathrm{CO}_{2}$ at $37^{\circ} \mathrm{C}$. The human umbilical vein cell line (EA.hy926 (ATCC ${ }^{\circledR}$ CRL-2922 ${ }^{\mathrm{TM}}$ )) and the human foreskin fibroblast cell line (HFF1 (SCSP-109, Stem Cell Bank, Chinese Academy of Sciences)) were cultured under humidified conditions in serum-free Dulbecco's modified Eagle’s medium (DMEM; Gibco; Invitrogen Pty Ltd., Australia) supplemented with 10\% FBS (ScienCell) and 1\% penicillin/streptomycin (P/S; ScienCell). Cells were cultured in a humidified $37^{\circ} \mathrm{C} / 5 \% \mathrm{CO}_{2}$ incubator. hBMSCs and HFF-1 cells were kindly provided by Stem Cell Bank, Chinese Academy of Sciences.

Separation and purification of small extracellular vesicles (sEVs): The sEVs secreted by BMSCs (B-sEVs) and sEVs secreted by miR-29b-3p-enriched BMSCs (B-miR29b-sEVs) were separated by ultracentrifugation. When the confluence of the human bone mesenchymal stem cell cultures reached $80 \%$, the culture medium was removed. The cells were then washed three times with PBS for 5 min each time. Then, the culture medium was replaced with serum-free medium. After $48 \mathrm{~h}$ of culture, the medium was collected and centrifuged at $300 \times \mathrm{g}$ for $10 \mathrm{~min}$ and $2000 \times \mathrm{g}$ for $15 \mathrm{~min}$ to remove dead cells and debris. The supernatant was ultracentrifuged two times at $100000 \times \mathrm{g}$ for $1.5 \mathrm{~h}$ each after aseptic filtration. Finally, the precipitates were resuspended in PBS.

Luciferase activity: Luciferase activity was assessed with a Dual-Luciferase Reporter Assay System (E1910, Promega, Madison, WI) according to the manufacturer's instructions. For the luciferase reporter assay, plasmids containing the wild-type or mutant 3'UTR of Col I were obtained from Obio Technology (Shanghai). Plasmids 
(200 ng/mL) and pre-miR-29b-3p or pre-miR-Control (20 nM) were cotransfected into the $293 \mathrm{~T}$ cell line. After $48 \mathrm{~h}$, luciferase levels were determined with the DualLuciferase Reporter Assay System (E1910, Promega) according to the manufacturer's instructions.

Synthesis of hydrogels. The photoinitiator I2959 (10 mg) was dissolved in $5 \mathrm{~mL}$ of deionized water. PEG diacrylate (PEG-DA; $0.1 \mathrm{~mL}, \mathrm{Mn}=250 / 700$ ) was then added to the solution, and the solution was stirred thoroughly again. Based on a method described in a previous study, the thiolated alginate (SA-SH) was synthesized from sodium alginate and cysteine methyl ester hydrochloride through amidation ${ }^{45}$. The above solution was then added to the sample bottle containing SA-SH powder (three different grafting rates-SA-SH-1: 19.71\%, SA-SH-2: 24.13\%, SA-SH -3: 31.35\%), and the sample was immediately irradiated under a UV lamp at $365 \mathrm{~nm}$ with rapid stirring for $10 \mathrm{~min}$. After UV irradiation, the SA-SH/PEG-DA hydrogel was removed and placed in a dialysis bag (MWCO=14 kDa) for dialysis to remove excess I2959 and PEG-DA that were not involved in the reaction. Six different types of SASH/PEG-DA hydrogels were prepared: SA-SH-1/PEG-DA-250, SA-SH-2/PEG-DA250, SA-SH-3/PEG-DA-250, SA-SH-1/PEG-DA-700, SA-SH-2/PEG-DA-700, and SA-SH-3/PEG-DA-700. According to the screening, SA-SH-3/PEG-DA-250 was selected as the lower layer of the hydrogel, and SA-SH-3/PEG-DA-700 was selected as the upper layer of the hydrogel.

Measurement of wound size reduction: Wounds were photographed with a digital camera (Canon, Japan) on days 0, 3, 7 and 14 after the operation. With ImageJ (National Institutes of Health, Bethesda, MD, USA), the margin of each wound and the marker were carefully traced, and the number of pixels encompassing each wound and marker was calculated. Wound closure was quantified using the following formula (1): Percent wound size reduction $=[(\mathrm{A} 0-\mathrm{At}) / \mathrm{A} 0] \times 100$, where $\mathrm{A} 0$ is the initial wound area $(\mathrm{t}=0)$ and At is the wound area at each time interval. 


\section{Supplementary figures}

A

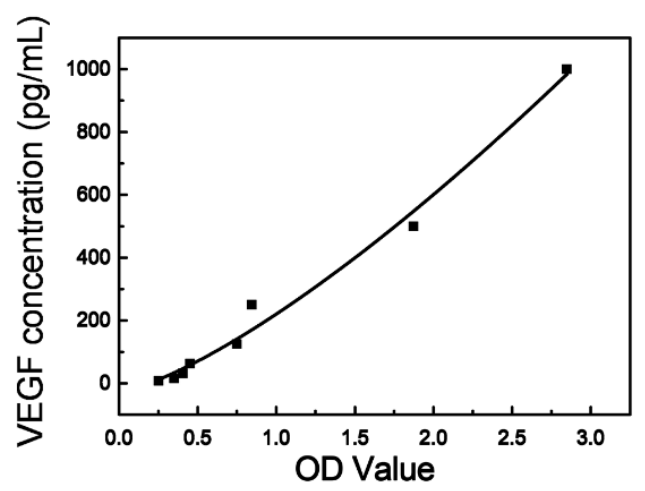

B

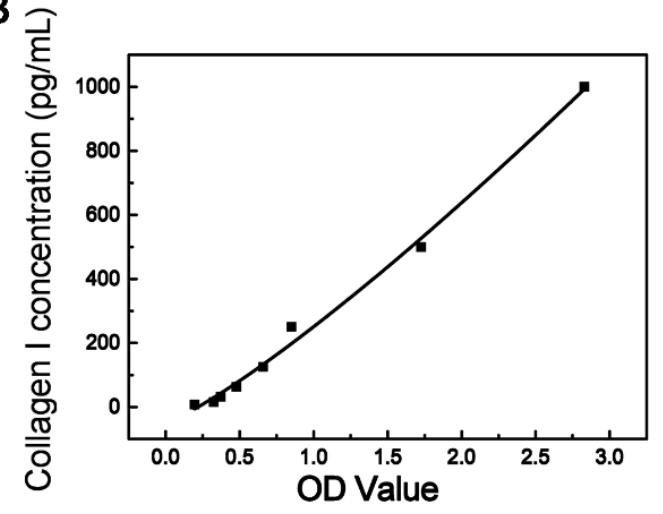

Figure S1. Standard curves for VEGF (A) and Col I (B) ELISA.

A
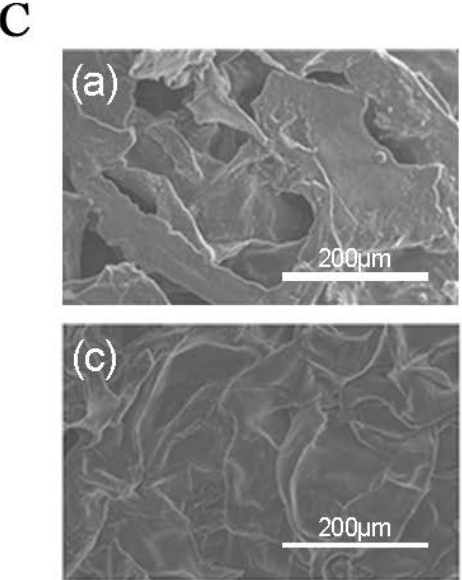


B

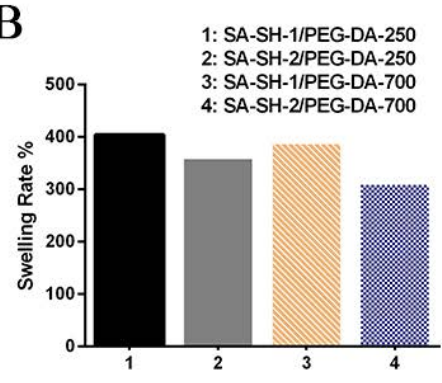

D1

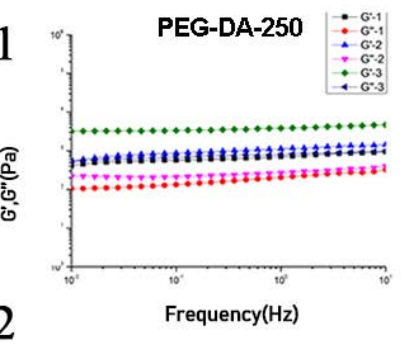

D2



Figure S2. (A) FTIR spectra of SA-SH/PEG-DA hydrogels. (B) Swelling rate of SASH/PEG-DA hydrogels. (C) Scanning electron micrographs of lyophilized SASH/PEG-DA hydrogels. (a) SA-SH-1/PEG-DA-250, (b) SA-SH-2/PEG-DA-250, (c) 
SA-SH-1/PEG-DA-700, (d) SA-SH-2/PEG-DA-700 (D1) Rheological behavior of SASH-3/PEG-DA-250 and (D2) SA-SH-3/PEG-DA-700 hydrogels.
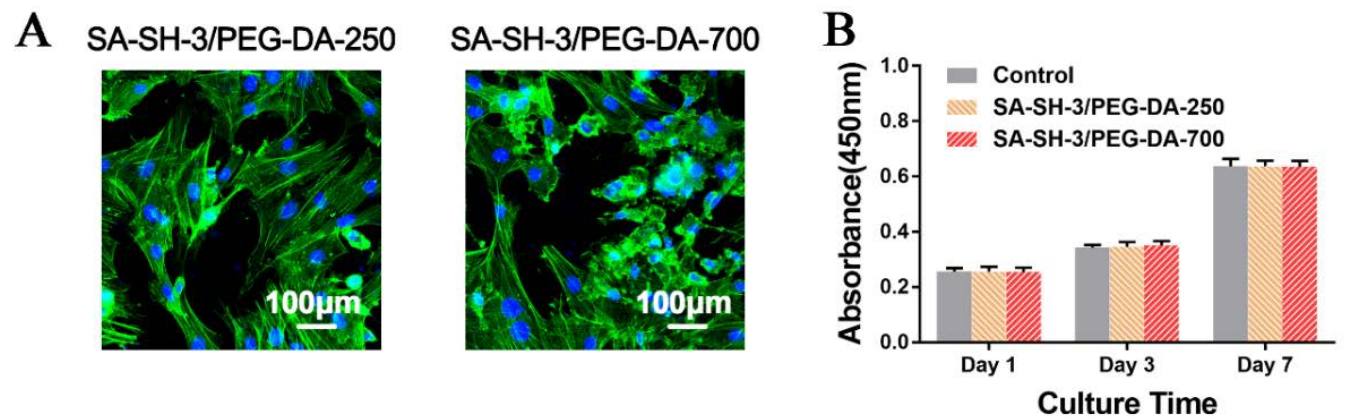

Figure S3. (A) Morphology of HFF-1 cells cultured on SA-SH-3/PEG-DA-250 and SA-SH-3/PEG-DA-700. (B) Viability of HFF-1 cells. 